\title{
Exploring diagenesis inanoxic sediments - R. Berner Lecture
}

\author{
ALEXANDRA (SASHA) TURCHYN ${ }^{1}$, ALEC MARTIN \\ HUTCHINGS $^{1}$ AND GILAD ANTLER ${ }^{2}$ \\ ${ }^{1}$ University of Cambridge \\ ${ }^{2}$ Ben-Gurion University of the Negev \\ Presenting Author: avt25@cam.ac.uk
}

Reconstructing changes in the Earth's redox state over the Proterozoic remains one of the outstanding goals of the geobiological and geological communities. Reconstruction of this redox evolution has been challenging, with many open questions as to the spatial and temporal extent of oxic, iron-rich (ferruginous) and sulfide-rich (euxinic) conditions. The relative extent of oxic, ferruginous, or euxinic oceans may provide answers to how changing environmental conditions influenced or shaped the evolution of multicellular life. What has been particularly enigmatic has been understanding the spatial extent of ocean euxinia, with various estimates ranging from 1 to $100 \%$ of the ocean floor underlaying a euxinic water column.

Many of the proxies for reconstructing redox evolution are hosted in shale-based lithologies, including the speciation of iron minerals, trace metal enrichments, and molybdenum isotope ratios. Here we will explore the last decade of our field research into East Anglian salt marsh sediments which are either ironrich, containing high concentrations of reduced iron, or sulfiderich, containing high concentrations of aqueous sulfide. We will show how the sediments in this field environment are initially iron-rich, but transform to sulfide-rich through a series of processes related to the balance of organic carbon versus electron acceptor availability within the environment. We argue that this environment, with deposition of large quantities of iron minerals, offers a unique opportunity to study how the exposure of ironrich sediments evolve under exposure to aqueous sulfide during early burial. We will show how exposure to sedimentary aqueous sulfide can overwrite and redistribute a primary geochemical signature and make the sediments look, mineralogically, geochemically, and isotopically, like those interpreted to have been deposited under a euxinic water column. We will demonstrate through measurements and modelling, as well as comparison with samples from the geological record, how this can impact the trace element concentration, molybdenum isotopic composition, and iron speciation. We suggest that shalebased proxies for euxinia are particularly troublesome given that iron-sulfur coupling in these environments leads to transformation of iron minerals and the diagenetic redistribution of metals. Our conclusion is that euxinia was far less prevalent in the Proterozoic than previous work suggests. 